\title{
Intervention Development Process for a Pragmatic Randomized Controlled Trial: The Thoracic Peri-Operative Integrative Surgical Care Evaluation Trial
}

\author{
Dugald Seely, ND, MSc, ${ }^{1-3}$ Julie E. Ennis, PhD, ${ }^{1,2}$ Ellen McDonell, ND, ${ }^{1,2}$ Anna Fazekas, MA, ${ }^{3}$ \\ Linlu Zhao, PhD, ${ }^{2}$ Tim Asmis, MD, Rebecca C. Auer, MD, MSc, ${ }^{3,4}$ Dean Fergusson, MHA, PhD, \\ Salmaan Kanji, PharmD, ${ }^{3,4}$ Donna E. Maziak, MD, MSc, ${ }^{4}$ Tim Ramsay, PhD, ${ }^{3}$ Paul Chamberland, $\mathrm{PT}^{4}$ \\ Chris Spooner, $\mathrm{ND}^{5}$ Jennifer Threader, $\mathrm{PhD}^{6}$ and Andrew Seely, MD, $\mathrm{PhD}^{3,4}$
}

\begin{abstract}
Background: Use of complementary therapies is high among people with cancer despite research gaps. The Thoracic Peri-Operative Integrative Surgical Care Evaluation (POISE) Trial will evaluate the impact of an integrative care intervention delivered by naturopathic doctors (NDs) in conjunction with usual care for patients undergoing surgery for lung, gastric, and esophageal cancer.

Objectives: To describe the multistep, multidisciplinary process of defining the integrative care intervention to be used in the Thoracic POISE trial using a principle-based approach that is pragmatic, holistic, safe, feasible, evidence driven, and consensus based.

Methods: An Intervention Development Committee (IDC) made up of a multidisciplinary team of health care providers (NDs, surgeons, oncologists, nurses, dietitians, physiotherapists, pharmacists, and psychologists), researchers, and patients was established to oversee the process. Potential intervention components were identified through a clinical practice survey and expert opinion. Systematic literature reviews were conducted and scores assigned based on the following criteria: usage, safety, goals, feasibility/scalability, and evidence. The IDC selected an intervention to be piloted that consists of a standard palette including core and optional components. Safety, known risks, and interactions with pharmaceuticals were evaluated using industry and professional monographs, a scoping literature review, and consultations with hospital pharmacists.

Results: The clinical practice survey and expert opinion identified 28 components for consideration. Following literature reviews, scoring, consensus from the IDC, and safety and interaction considerations, an intervention palette consisting of core and optional components was defined. The intervention options vary based on the patient's phase of treatment and symptom-specific needs. The intervention includes supplements, physical recommendations (exercise), nutritional counseling, and psychological support (audio scripts).

Conclusion: Through a multistep, multidisciplinary process an integrative care intervention was developed for the Thoracic POISE trial. The intervention will be piloted in a single-arm feasibility study, followed by a single-center randomized controlled trial (RCT), and finally a multicenter RCT.
\end{abstract}

\footnotetext{
${ }^{1}$ Canadian College of Naturopathic Medicine, Toronto, Canada.

${ }^{2}$ Ottawa Integrative Cancer Centre, Ottawa, Canada.

${ }^{3}$ Clinical Epidemiology Program, Ottawa Hospital Research Institute, Ottawa, Canada.

${ }^{4}$ Ottawa Hospital, General Campus, Ottawa, Canada.

${ }^{5}$ Paradigm Naturopathic Medicine, Vernon, Canada.

${ }^{6}$ The Royal Melbourne Hospital, Melbourne, Australia.
} 
Keywords: integrative medicine, naturopathic medicine, thoracic cancer, surgery, quality of life, natural health products, lifestyle management

\section{Introduction}

$\mathbf{T}$ HORACIC CANCERS ARE THE LEADING cause of cancerrelated death in North America. For early-stage cancers, surgical resection is the mainstay of therapy with intent to cure. $^{1,2}$ Although potentially life-saving, thoracic cancer surgery poses two critical issues: minor and major adverse events in the perioperative period, ${ }^{3-5}$ and the risk of recurrence in the months to years after surgery. ${ }^{6-8}$ As a result, both the diagnosis of thoracic cancer and its management may impair quality of life (QoL) ${ }^{9}$ and lead to heavy utilization of health care. ${ }^{10-12}$ Efforts to improve QoL for patients with thoracic malignancy, reduce their health care costs, and improve their short- and long-term outcomes are of great importance.

Integrative oncology incorporates evidence-based complementary medicine with conventional medicine using a coordinated holistic approach and comprises the use of natural health products (NHPs; e.g., herbal formulas, vitamins, and minerals), physical therapy interventions (e.g., physiotherapy and massage), psychological support (e.g., counseling and meditation), and lifestyle behavior changes (e.g., nutrition and physical activity) supported by a growing body of evidence. ${ }^{13}$

More than half of adults diagnosed with cancer worldwide report the use of these interventions. ${ }^{14}$ However, these complementary practices are often self-directed, without clear guidance from their family doctor, surgeon, oncologist, dietitian, naturopathic doctor (ND), or other health care provider. The high level of patient interest, compelling preclinical ${ }^{15}$ and clinical studies $^{16-18}$ have prompted further research into the safety and efficacy of integrative therapies. Existing clinical trials in integrative medicine tend to focus on single-agent therapies, rather than the holistic, multifaceted, and individualized therapies that are commonly used in real-life settings.

To address this gap, we are conducting a pragmatic randomized controlled trial (RCT) in thoracic (lung, esophageal, and gastric) cancer patients - the Thoracic Peri-Operative Integrative Surgical Care Evaluation (POISE) trial-designed to evaluate the impact of integrative care delivered by NDs alongside usual care. In the first stage of the Thoracic POISE trial we defined a standardized, multimodal palette of complementary care interventions within the practice scope of NDs. The palette was developed through a principle-driven process requiring evidence suggesting safety in thoracic cancer populations. The objectives of this article are to describe the evidence-based, collaborative, multidisciplinary process of selecting the elements to be included in an integrative care intervention, through the process of evaluating, ranking, and selecting our intervention palette.

\section{Methods}

\section{Overview of trial}

The Thoracic POISE trial is a three-stage clinical research project following an adaptive design that culminates in a multicenter two-arm parallel-design RCT with a total planned enrollment of 440 participants. The RCT compares an integrative care intervention (consisting of supplemental [NHPs], physical, psychological, and nutritional therapies) delivered by NDs over the course of 1 year alongside hospital-based care to usual hospital-based care alone.

Before embarking on the multicenter RCT, the intervention and study protocol will be piloted in a single-arm feasibility study beginning in the fall of 2018. Specifically, the research team aims to pilot the ability to recruit eligible patients, the compliance of participants with the integrative care intervention, and compliance to and ease of scheduling outcome assessments (i.e., questionnaires, blood draws, and studyrelated visits). The feasibility study will be followed by a single-center two-arm parallel-design RCT to commence in 2020 followed by multicenter enrollment soon thereafter.

Patients will be approached for consent for the integrative care intervention once they have been deemed eligible for thoracic surgical resection of a lung or gastroesophageal malignancy with curative intent. Dependent on the stage of the underlying cancer, as part of usual care, patients may be offered neoadjuvant or postoperative chemo- and/or radiation therapy, or both. The group allocated to the integrative care arm will receive the intervention over two periods, first preoperatively between consent and surgery, and second, postoperatively, from the end of hospitalization through to the end of the first year postsurgery.

The primary outcome for the RCT is QoL measured over the first year of recovery; major secondary outcomes include intra- and postoperative adverse events, overall survival, and cost effectiveness. Additional objectives include determination of biological impact of the interventions on immune and inflammatory function, and evaluation of qualitative outcomes within a rigorous mixed-methods approach. The Canadian Association of Thoracic Surgery (CATS) in partnership with the Ottawa Integrative Cancer Center (OICC) will collaborate to accomplish this trial.

\section{Intervention selection principles}

Thoracic POISE seeks to answer the question of whether integrating care provided by NDs alongside usual care improves outcomes in thoracic cancer patients eligible for curative intent surgery. To enhance the generalizability of the findings, we sought to develop a standardized palette of therapies to be administered during the trial. A core set of principles were first established to guide the selection process to include components that are: (1) pragmatic: reflect common clinical usage among NDs who focus on cancer care; (2) holistic: include four domains: supplemental, physical, psychological, and nutritional and address multiple therapeutic goals (e.g., anti-inflammatory, immunostimulatory); (3) evidence driven: show evidence of benefit and safety in scientific research; (4) safe: do not create significant side effects or interfere with conventional care; (5) feasible: can be delivered by NDs in time allowed with adequate patient adherence and compliance and operationalized to allow replication in other settings; and (6) 
multidisciplinary consensus based: selected by consensus among a multidisciplinary group of clinicians, practitioners, researchers, and patients.

\section{Intervention Development Committee: roles and responsibilities}

An Intervention Development Committee (IDC) made up of a broad team of health care providers (including NDs, surgeons, oncologists, dietitians, nurses, physiotherapists, pharmacists, and psychologists), epidemiologists, and patients from across Canada and internationally was established (Supplementary Data) to oversee the intervention development process (Fig. 1). IDC members with relevant clinical expertise were selected as Domain Leads within each of the four intervention domains (Supplemental: ND [C.S.], Physical: physiotherapist [P.C.], Psychological: psychologist [J.T.], Nutritional: ND [C.H.]). The IDC was responsible for guiding the development of the intervention, ratifying the intervention selection process, and assuming responsibility for the intervention palette selected for the study. The Domain Leads were responsible for suggesting intervention components, reviewing evidence, scoring intervention components, and presenting the assessments to the IDC to inform their decision making for the selection of the intervention. The IDC will be responsible for revising the intervention palette based on the single-arm Stage I feasibility study. Research team members (J.E., E.M., and L.Z.) were responsible for overseeing the conduct and synthesis of the evidence summaries.

\section{Identification of potential intervention components}

The primary approach to identifying potential components for inclusion in the intervention palette was a naturopathic oncology practice review described elsewhere. ${ }^{19}$ Briefly, members of the Oncology Association of Naturopathic Physicians (OncANP), a professional association of NDs who work with people living with cancer were invited to complete an online survey designed to collect information on types, therapeutic goals, and contraindications of therapies considered for use in the pre- and postoperative care of patients with thoracic cancer. Details on the administration of each therapy (dose, dosage regimen, treatment duration, and route of administration) were collected in the survey to help the IDC assess feasibility and scalability of interventions. Forty-four NDs responded, all of whom were trained and practice in North America. Additional components not identified from the survey were considered for inclusion based on expert opinion and evidence review.

\section{Evidence review and synthesis}

Evidence reviews were performed for the integrative therapies identified by $\geq 40 \%$ of survey respondents in the naturopathic oncology practice survey. Four literature databases (MEDLINE, Embase, Cochrane Library, and Allied and Complementary Medicine Database) were systematically searched using a modified version of PubMed's Complementary Medicine Subset Strategy ${ }^{20}$ with general cancer and surgery search vocabulary (Supplementary Data). Publications were screened for inclusion based on the following criteria: (1) Human clinical trial results (controlled or noncontrolled) or systematic review/meta-analysis of human clinical trials; (2) Investigate one of the integrative therapies of interest; (3) Thoracic (lung, gastric, and/or esophageal) cancer treatment investigated as the primary focus of the trial for original articles or general cancer for systematic reviews/meta-analyses; and (4) English language. Domain leads (C.H., P.C., J.T., and C.P.) conducted scoping reviews to identify additional literature in other cancers and in other surgical procedures as supplementary evidence.

Recognizing their importance in the field of nutritional science and the relative scarcity of clinical trial data, the inclusion criteria were expanded to include observational studies for the nutritional domain. ${ }^{21}$ Relevant details were extracted by research staff (E.M., J.E., and L.Z.) from each study including design, population, intervention, control, outcomes, side effects, and interactions. Evidence summaries for each of the integrative therapy components presented information on the number and types of studies showing benefit, no effect, or harmful effect for each outcome reported and a summary of the evidence related to safety and adverse effects.
FIG. 1. Flow diagram of the Thoracic POISE trial intervention development process. IDC, Intervention Development Committee; ND, Doctor of Naturopathic Medicine; POISE, PeriOperative Integrative Surgical Care Evaluation.

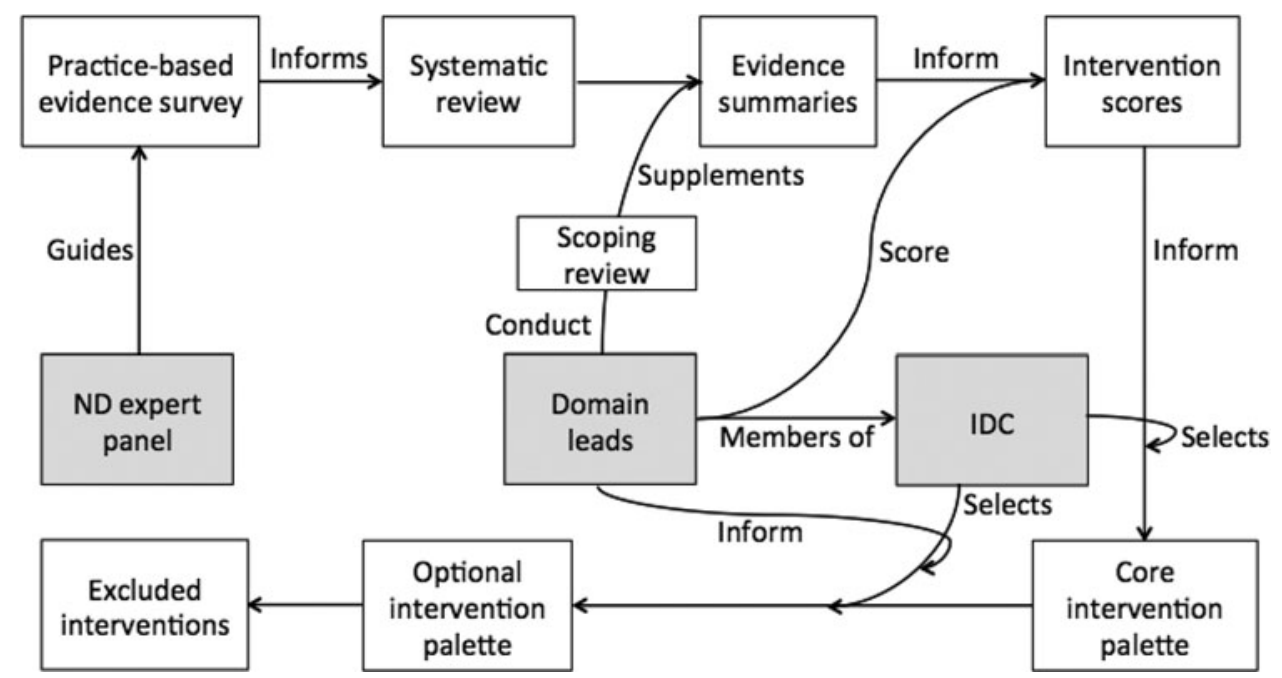




\section{Score assignment and intervention selection}

To facilitate the intervention selection process, we developed a scoring system to rank potential components. Each of the top identified integrative therapies within the pre- and postoperative settings were independently scored by the relevant domain lead and by research staff (J.E. and E.M.) according to five of the intervention selection principles (pragmatic, holistic, evidence-driven, safe, and feasible and scalable; Table 1). This scoring system is a composite consisting of objective evaluations of (1) practice usage and (2) evidence from the medical literature, subjective evaluations of (3) safety, (4) feasibility and scalability, and a mixed subjective and objective evaluation of (5) therapeutic goals. Each of the five areas were assigned a score from 1 to 5 except for (2) the evidence score that was assigned a higher weight with a score from 1 to 10 . The total composite scores thus ranged from 5 to 30, with a higher score indicating a better overall representation of the intervention selection principles.

Details of the scoring criteria are outlined in Table 1. Scores were assigned for use in the preoperative period considering evidence for surgical complications and for use in the postoperative, long-term maintenance period considering evidence for survival, QoL, and other relevant biological outcomes. The independently generated scores were compared and discrepancies were resolved by discussion including the Principal Investigators (Dugald Seely, ND, MSc, Andrew Seely, MD, PhD, FRCSC). As the final step, the IDC convened at an Intervention Selection Meeting to review and discuss the evidence summaries and scores for each therapy and make a final determination on the intervention palette to be pilot tested in the single-arm feasibility stage.

\section{Safety and interactions review}

The potential for negative interactions between conventional cancer therapies and integrative therapies, especially NHPs, is an important concern for both clinicians and patients. ${ }^{22}$ A focused evidence review was conducted in consultation with trial pharmacists (S.K. and L.R.) to evaluate the potential for harm posed by the NHPs selected for inclusion in the intervention palette. Sources reviewed included NHP product monographs produced by Health Canada $^{23}$ and the Natural Medicines Comprehensive Database, which aggregates and synthesizes data on complementary and alternative medicine.

The significance of interactions is evaluated using a StopLight Rating System combining information on occurrence and severity. ${ }^{24}$ Trial pharmacists reviewed all interactions rated as "Major" (do not use combination) or "Moderate" (use cautiously or avoid combination) and having an occurrence rating of at least "Possible" (evidence suggests interaction might occur in some patients) and a severity rating of at least "Moderate" (moderate impairment or significant discomfort possible). Based on this evaluation, absolute and relative contraindications were determined and documented in the trial procedures manual and were used to guide the periods in which intervention components are to be applied in the trial.

\section{Results}

The systematic and multidisciplinary process followed resulted in a palette of integrative interventions that are pragmatic, holistic, evidence driven, safe, feasible and scalable, and importantly agreed upon by the multidisciplinary stakeholders directly involved in thoracic cancer care.

\section{Intervention component screening}

The results of the intervention screening processes and database search results are given in Figure 2. The naturopathic practice survey identified 23 components for consideration (14 supplemental, 2 physical, 4 psychological, and 3 nutritional). Five additional components were brought forward by Domain Leads and other IDC members (psychoeducation, inspiratory muscle training, mistletoe, L-arginine, yoga). The evidence generated by the systematic review resulted in variable literature availability across components. Therapies with the most literature available were omega-3 (fish oil; 11 systematic review/meta-analyses and 26 clinical trials) and exercise therapy (40 systematic review/meta-analyses and 36 clinical trials). For several components including arnica, bromelain, modified citrus pectin, whey protein, and diaphragmatic breathing, no records were identified in the database search that met the inclusion criteria. No clinical trials were available for specific dietary approaches (e.g., Mediterranean diet, low glycemic index diet); however, data from observational studies were available and considered. Despite being identified as potential components, yoga, acupuncture, and intravenous administration of any therapies were deemed infeasible given the requirement for practitioner administration; the intervention was being developed to allow for use at home by patients. Therefore, no further evidence review was conducted for these therapies.

\section{Intervention component scoring}

The scores assigned to the components considered for inclusion in the pre- and postoperative settings are given in Table 2. The generation of the score for Coriolus versicolor in the postoperative setting is provided as an example in Table 1. Of a possible 30 points, therapies earned scores ranging from 14 (modified citrus pectin-preoperative) to 26 (Coriolus versicolor-postoperative). The highest scoring preoperative components were probiotics (24), melatonin (23), fish oil (22), and exercise therapy (22), whereas Coriolus versicolor (26), astragalus (24), curcumin (23), fish oil (23), melatonin (23), and exercise therapy (22) received the highest scores in the postoperative setting.

\section{Intervention design}

The IDC realized the need for differentiating the intervention with respect to individual patients' phases of treatment, and the need to balance standardization and individual patient customization. To address the first issue, the intervention was reframed into four distinct phases based on the periods of usual care study participants may experience: (1) neoadjuvant, (2) perioperative $(\sim 4$ weeks before and after surgery), (3) adjuvant, and (4) maintenance. Second, it was recommended that the intervention be further framed as a core palette of standardized components to be recommended to all study participants with an optional palette of components to be recommended based on specific side effects of chemotherapy and radiotherapy. Since the methods for 
Table 1. Component Scoring Criteria for the Facilitation of the Integrative Intervention Palette Selection Process by the Thoracic POISE Trial Intervention Development Committee

\begin{tabular}{|c|c|c|c|c|}
\hline \multicolumn{3}{|c|}{ Intervention score } & \multicolumn{2}{|c|}{$\begin{array}{c}\text { Example } \\
\text { Domain: Natural health products } \\
\text { Intervention: Coriolus versicolor } \\
\text { (postoperative) }\end{array}$} \\
\hline Principle & Scoring criteria & Scoring range & $\begin{array}{l}\text { Scoring rationale and } \\
\text { commentary }\end{array}$ & Score \\
\hline Pragmatic & $\begin{array}{l}\text { Objective score based on } \\
\text { practice-based survey results } \\
\text { scored as follows: } \\
\begin{aligned} 5=>85 \% \\
4=70-85 \% \\
3=55-69 \% \\
2=40-54 \% \\
1=<40 \%\end{aligned}\end{array}$ & $1-5$ & $\begin{array}{l}\text { Survey indicates } 43 \% \\
\text { recommended }\end{array}$ & 2 \\
\hline Holistic & $\begin{array}{l}\text { Mixed score based on practice- } \\
\text { based survey responses and } \\
\text { inclusions from domain lead } \\
\text { scored as follows: } \\
1 \text { point for every therapeutic } \\
\text { goal identified by } \geq 50 \% \text { of } \\
\text { practice-based survey } \\
\text { respondents and domain lead } \\
\text { up to a maximum of } 5\end{array}$ & $1-5$ & $\begin{array}{l}\text { Augment immune function } \\
(90 \%) ; \text { antineoplastic } \\
(74 \%) ; \text { and enhance } \\
\text { health-related quality } \\
\text { of life }(32 \%)\end{array}$ & 3 \\
\hline Evidence driven & $\begin{array}{l}\text { Objective score based on literature } \\
\text { evidence for benefit in thoracic } \\
\text { cancer, thoracic surgery, and } \\
\text { solid tumors scored as follows: } \\
9-10=\text { Systematic review and } \\
\text { meta-analysis including } \\
2+\text { RCTs with benefit } \\
7-8=\text { At least one RCT } \\
\text { demonstrates benefit in } \\
\text { absence of negative findings } \\
5-6=\text { Non-RCTs with evidence of } \\
\text { benefit } \\
3-4=\text { Cohort and/or case-control } \\
\text { studies demonstrating benefit } \\
1-2=\text { Evidence from preclinical } \\
\text { and case reports only }\end{array}$ & $1-10$ & $\begin{array}{l}\text { Systematic review of } 11 \\
\text { RCTs/non-RCTs for lung } \\
\text { cancer } \\
\text { Meta-analysis of } 13 \text { RCTs in } \\
\text { mixed cancer (including } \\
\text { gastric and esophageal) } \\
1 \text { RCT in NSCLC } \\
\text { Strong evidence for improved } \\
\text { survival and quality of life } \\
\text { with Coriolus versicolor } \\
\text { alongside conventional } \\
\text { treatment }\end{array}$ & 9 \\
\hline Safe & $\begin{array}{l}\text { Subjective score based on evidence } \\
\text { of harm from literature, survey } \\
\text { findings, likelihood of } \\
\text { interactions, and adverse effects } \\
\text { assessed by domain lead as } \\
\text { follows: } \\
5=\text { Lowest likelihood for possible } \\
\text { harm } \\
1=\text { Highest likelihood for } \\
\text { possible harm }\end{array}$ & $1-5$ & $\begin{array}{l}\text { Safe with appropriate oral } \\
\text { use, with no known } \\
\text { interactions }\end{array}$ & 5 \\
\hline Feasible and scalable & $\begin{array}{l}\text { Subjective score based on two key } \\
\text { aspects assessed by domain lead } \\
\text { as follows: } \\
\text { Ability for patients to adopt } \\
\text { recommendations; and ability } \\
\text { to operationalize the } \\
\text { intervention and transferability } \\
\text { to another ND }\end{array}$ & $1-5$ & $\begin{array}{l}\text { Oral capsule } 1.5 \mathrm{~g} \text { b.i.d. } \\
\text { without food }\end{array}$ & 5 \\
\hline Total & & $5-30$ & & 26 \\
\hline
\end{tabular}

ND, naturopathic doctor; NSCLC, nonsmall cell lung cancer; POISE, Peri-Operative Integrative Surgical Care Evaluation; RCT, randomized controlled trial. 

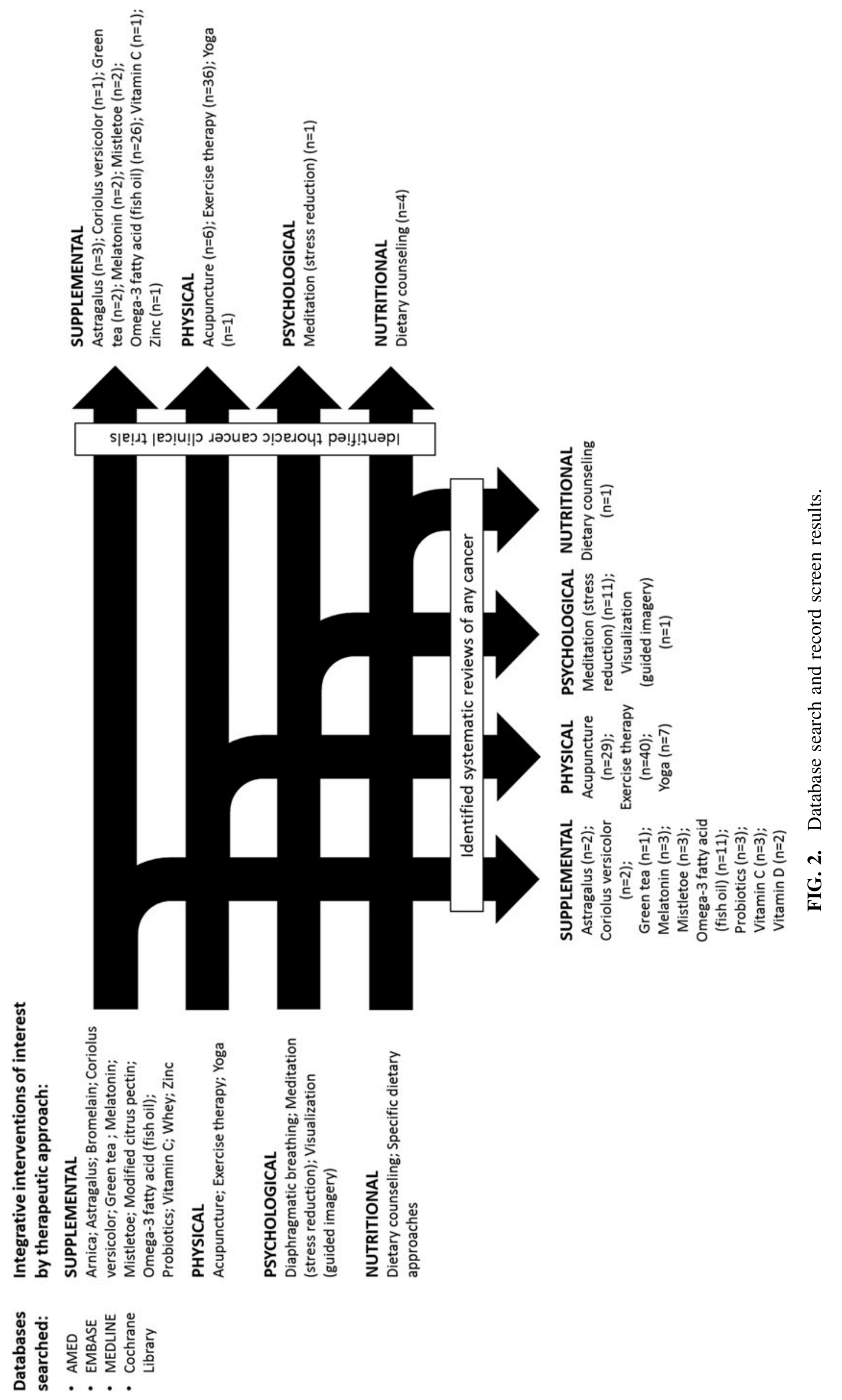
Table 2. Score Assignment for Integrative Intervention Components Considered for Thoracic POISE Trial

\begin{tabular}{|c|c|c|c|c|c|c|}
\hline & $\begin{array}{l}\text { Practical } \\
\text { usage }\end{array}$ & Safety & $\begin{array}{c}\text { Holism } \\
\text { across goals }\end{array}$ & $\begin{array}{c}\text { Feasibility } \\
\text { and scalability }\end{array}$ & $\begin{array}{c}\text { Evidence } \\
\text { for effectiveness }\end{array}$ & $\begin{array}{l}\text { Total } \\
\text { score }\end{array}$ \\
\hline \multicolumn{7}{|l|}{ Preoperative setting } \\
\hline \multicolumn{7}{|l|}{ Supplemental } \\
\hline Probiotics & 2 & 4 & 4 & 5 & 9 & 24 \\
\hline Melatonin & 1 & 4 & 3 & 5 & 10 & 23 \\
\hline Fish oil & 2 & 4 & 5 & 4 & 7 & 22 \\
\hline Arnica & 4 & 3 & 4 & 5 & 5 & 21 \\
\hline L-Arginine & 1 & 4 & 3 & 4 & 9 & 21 \\
\hline Vitamin D & 3 & 4 & 4 & 3 & 4 & 18 \\
\hline Zinc & 2 & 4 & 3 & 5 & 4 & 18 \\
\hline Bromelain & 2 & 3 & 4 & 4 & 3 & 16 \\
\hline Whey & 2 & 5 & 3 & 4 & 2 & 16 \\
\hline Mistletoe & 1 & 3 & 3 & 3 & $\overline{5}$ & 15 \\
\hline Modified citrus pectin & 4 & 3 & 1 & 3 & 3 & 14 \\
\hline \multicolumn{7}{|l|}{ Physical } \\
\hline Exercise therapy & 3 & 4 & 5 & 3 & 7 & 22 \\
\hline Inspiratory muscle training & 1 & 4 & 3 & 3 & 7 & 18 \\
\hline \multicolumn{7}{|l|}{ Psychological } \\
\hline Diaphragmatic breathing & 2 & 4 & 3 & 4 & 7 & 20 \\
\hline Visualization/guided imagery & 2 & 4 & 3 & 3 & 7 & 19 \\
\hline Meditation & 3 & 4 & 4 & 3 & 4 & 18 \\
\hline Psychoeducation & 1 & 4 & 2 & 3 & 7 & 17 \\
\hline Stress reduction & 3 & 4 & 4 & 2 & 3 & 16 \\
\hline \multicolumn{7}{|l|}{ Nutritional } \\
\hline Mediterranean diet & 2 & 5 & 3 & 3 & 4 & 17 \\
\hline Anti-inflammatory diet & 1 & 5 & 5 & 2 & 4 & 17 \\
\hline Low glycemic index diet & 2 & 5 & 3 & 3 & 3 & 16 \\
\hline \multicolumn{7}{|l|}{ Postoperative setting } \\
\hline \multicolumn{7}{|l|}{ Supplemental } \\
\hline Coriolus versicolor & 2 & 5 & 5 & 5 & 9 & 26 \\
\hline Astragalus & 2 & 4 & 4 & 5 & 9 & 24 \\
\hline Curcumin & 3 & 4 & 4 & 5 & 7 & 23 \\
\hline Fish oil & 4 & 4 & 4 & 4 & 7 & 23 \\
\hline Melatonin & 2 & 4 & 3 & 5 & 9 & 23 \\
\hline Vitamin D & 3 & 4 & 4 & 3 & 7 & 21 \\
\hline Mistletoe & 1 & 3 & 3 & 3 & 8 & 18 \\
\hline Green tea & 2 & 3 & 3 & 5 & 4 & 17 \\
\hline Probiotics & 2 & 4 & 4 & 5 & 0 & 15 \\
\hline Whey & 2 & 5 & 3 & 4 & 1 & 15 \\
\hline \multicolumn{7}{|l|}{ Physical } \\
\hline Exercise therapy & 3 & 4 & 5 & 3 & 7 & 22 \\
\hline \multicolumn{7}{|l|}{ Psychological } \\
\hline Meditation & 3 & 4 & 4 & 2 & 8 & 21 \\
\hline Stress reduction & 3 & 4 & 4 & 2 & 6 & 19 \\
\hline Diaphragmatic breathing & 2 & 4 & 3 & 4 & 2 & 15 \\
\hline Visualization/guided imagery & 1 & 4 & 4 & 3 & 2 & 14 \\
\hline \multicolumn{7}{|l|}{ Nutritional } \\
\hline Mediterranean diet & 2 & 5 & 3 & 3 & 4 & 17 \\
\hline Anti-inflammatory diet & 1 & 5 & 5 & 2 & 4 & 17 \\
\hline Low glycemic index diet & 2 & 5 & 3 & 3 & 3 & 16 \\
\hline
\end{tabular}

POISE, Peri-Operative Integrative Surgical Care Evaluation.

identifying and reviewing intervention components were not designed considering chemotherapy and radiotherapy side effect management as outcomes, the NDs serving as voting members on the IDC (C.H., C.S., D.S., L.A., and M.T.) were asked to identify any additional therapies they would use to manage the most common, clinically significant side effects. Evidence was reviewed for therapies recommended by at least two ND IDC members.

\section{Intervention selection}

The components for inclusion in the integrative care intervention were finalized by the IDC as follows. The final recommendations for the (1) supplemental domain are given in Table 3. Vitamin D, melatonin, Coriolus versicolor, and fish oil will be included as core components throughout all phases. Other core palette components are phase 


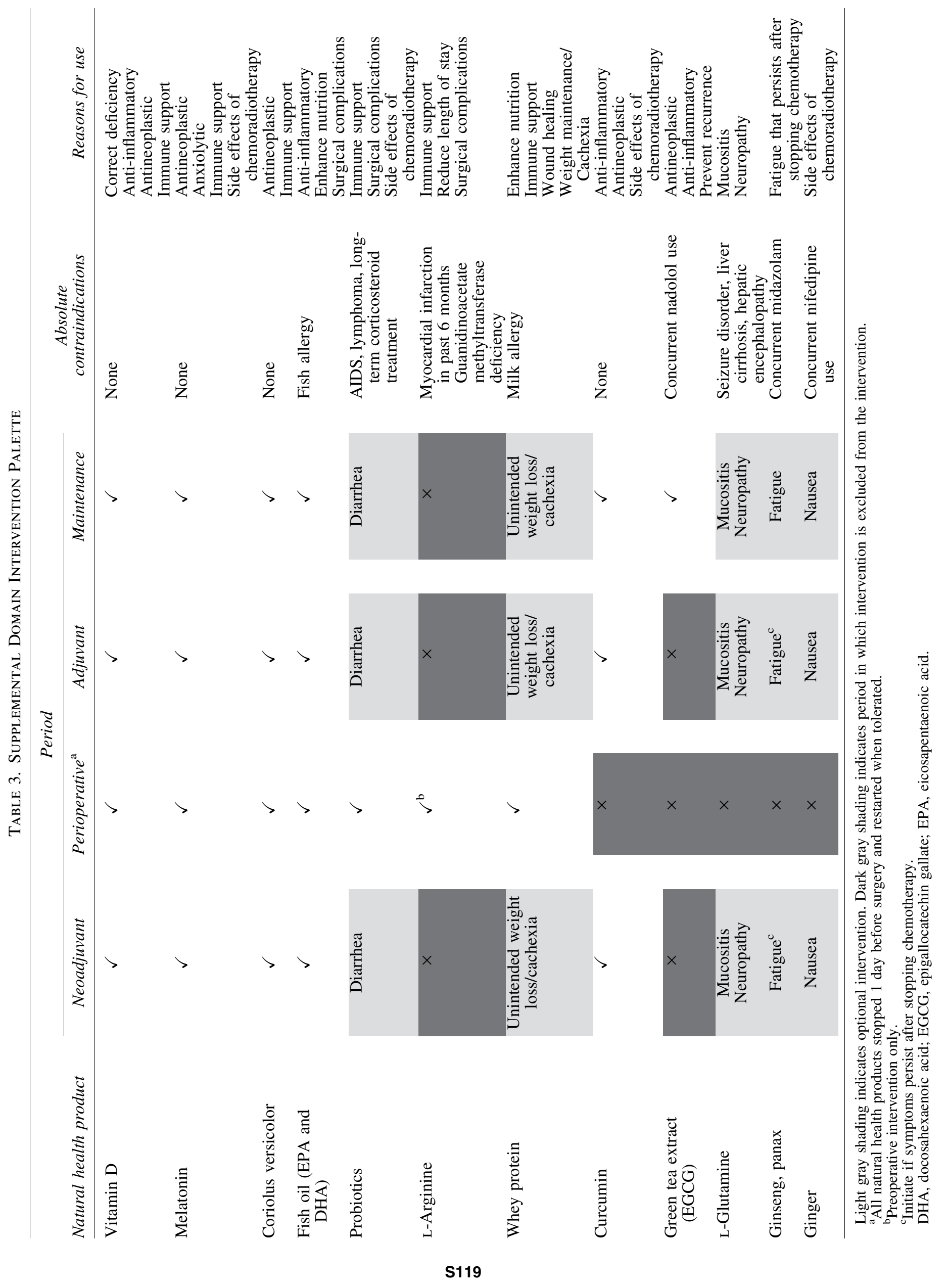


Table 4. Behavioral Intervention Palette

\begin{tabular}{|c|c|c|c|}
\hline Domain & Intervention & Description & Reasons for use \\
\hline Nutritional & $\begin{array}{l}\text { Nutritional } \\
\text { counseling }\end{array}$ & $\begin{array}{l}\text { ND-led counseling individualized to patients' current diet, } \\
\text { self-efficacy, and clinical status. } \\
\text { In neoadjuvant, perioperative, and adjuvant periods } \\
\text { counseling will emphasize the importance of ensuring } \\
\text { adequate calorie and protein intake. } \\
\text { Counseling in the maintenance period will emphasize an } \\
\text { overall healthful dietary pattern with a base } \\
\text { Mediterranean approach. The Mediterranean diet focuses } \\
\text { on: } \\
\text { Eating primarily plant-based foods (fruits and vegetables, } \\
\text { whole grains, legumes, and nuts) } \\
\text { Eating fish and poultry at least twice a week } \\
\text { Limiting red meat to no more than a few times a month } \\
\text { Using healthy fats, herbs, and spices. } \\
\text { Targeted recommendations will be made for foods } \\
\text { suggested to have anticancer properties, low glycemic } \\
\text { index foods, and anti-inflammatory foods } \\
\text { Recipes, sample menu plans, and approaches to modify food } \\
\text { texture will be provided }\end{array}$ & $\begin{array}{l}\text { Anti-inflammatory } \\
\text { Enhance nutrition } \\
\text { Weight management } \\
\text { Glycemic control }\end{array}$ \\
\hline Physical & Exercise & $\begin{array}{l}\text { ND to prescribe } 30+\text { min moderate intensity aerobic } \\
\text { exercise plus resistance exercises; } 5 \text { days/week } \\
\text { Walking is the recommended aerobic exercise at target is } \\
\text { RPE 12-14 } \\
\text { ND to provide document outlining intensity levels (Borg } \\
\text { RPE scale) for walking } \\
\text { Duration can be modified based on base physical fitness of } \\
\text { patient and clinical status } \\
\text { Postoperative walking to be resumed postdischarge } \\
\text { Resistance training: program of six exercises } \\
\text { ND to demonstrate exercises and provide document } \\
\text { explaining resistance increments for exercises. Lower } \\
\text { body uses body weight, upper body uses } 1-2 \text { kg weights } \\
\text { Postoperative patients to resume lower body exercises, } \\
\text { but wait until } 6 \text { weeks postoperative to resume upper } \\
\text { body) } \\
\text { Patients instructed to keep an exercise diary } \\
\text { Exercise prescription modified as needed for contraindicated } \\
\text { conditions including: uncontrolled hypertension; severe } \\
\text { limiting MSK conditions, cardiac/pulmonary disease, } \\
\text { chest wall or joint pain or psychiatric illness } \\
\text { Dealing with diverse population so general } \\
\text { recommendations to encourage adherence include: (1) any } \\
\text { activity is better than no activity; ( } 2 \text { ) try to achieve } 30 \text { min } \\
\text { of continuous activity daily; (3) try to achieve moderate } \\
\text { intensity }\end{array}$ & $\begin{array}{l}\text { Antineoplastic } \\
\text { Anxiety } \\
\text { Cardiopulmonary fitness } \\
\text { Fatigue } \\
\text { Immune support } \\
\text { Prevent recurrence } \\
\text { Reduce inflammation } \\
\text { Surgical complications } \\
\text { Wound healing }\end{array}$ \\
\hline & IMT & $\begin{array}{l}\text { Preoperative intervention for participants with impaired } \\
\text { pulmonary function (FEV1<60\% [lung] or }<75 \% \\
\text { [esophageal], gastric) } \\
\text { ND to explain IMT to eligible patients and assess for MIP } \\
\text { IMT recommendation: } 1 \times 15 \text { min session/day, } 7 \text { days/week, } \\
15 \text { min/session until surgery; intensity: starting at } 30 \% \text { of } \\
\text { MIP and increasing by } 2 \mathrm{~cm} \mathrm{H}_{2} \mathrm{O} \text { weekly up to max of } \\
60 \% \text { MIP } \\
\text { Contraindication: untreated pneumothorax }\end{array}$ & $\begin{array}{l}\text { Postoperative lung } \\
\text { function recovery } \\
\text { Reduce length of stay } \\
\text { Surgical complications }\end{array}$ \\
\hline Psychological & $\begin{array}{l}\text { Mind-body } \\
\text { medicine audio } \\
\text { recordings }\end{array}$ & $\begin{array}{l}\text { ND to administer audio recordings with psychoeducation, } \\
\text { visualization, breathing exercises, mindfulness, gentle } \\
\text { movement, and meditation }\end{array}$ & $\begin{array}{l}\text { Anxiety } \\
\text { Immune support } \\
\text { Pain }\end{array}$ \\
\hline
\end{tabular}

IMT, inspiratory muscle training; MIP, max inspiratory pressure; MSK, musculoskeletal; ND, naturopathic doctor; RPE, rate of perceived effort. 
dependent and include curcumin (neoadjuvant, adjuvant, and maintenance), probiotics (perioperative), whey protein (perioperative), L-arginine (preoperative), and green tea extract (maintenance). Optional palette components for the neoadjuvant, adjuvant, and maintenance periods include probiotics (diarrhea), whey protein (unintended weight loss/cachexia), L-glutamine (mucositis, neuropathy), panax ginseng (fatigue), and ginger (nausea).

For the behavioral domains (i.e., nutritional, psychological, and physical) the IDC recommended that resources be developed and counseling be provided to support participants to make healthy lifestyle changes (Table 4). The (2) nutritional domain will include general counseling promoting a healthy dietary pattern based on the Mediterranean diet. Participants will be encouraged to consume primarily plant-based foods, fish, and poultry at least twice a week and to use healthy fats, herbs, and spices and will be encouraged to limit red and processed meat and alcohol consumption. Aspects of other dietary approaches will be considered within the context of the Mediterranean diet (i.e., antiinflammatory, low glycemic index). Targeted recommendations will be made for specific foods (e.g., cruciferous vegetables, fermented foods, lower glycemic index foods).

During active treatment (neoadjuvant, perioperative, and adjuvant), counseling will emphasize the importance of ensuring caloric sufficiency $(30-35 \mathrm{kcal} / \mathrm{kg}$ ideal or corrected body weight) and adequate protein intake (1.2-1.5 g/kg ideal or corrected body weight). Resources to support adherence to dietary recommendations will be provided including an outline of the nutritional recommendations, sample meal ideas, and tips for making dietary changes. For the (3) psychological domain, all the therapies considered (i.e., psychoeducation, visualization, diaphragmatic breathing exercises, and mindfulness meditation) will be made available as audio recordings. Study NDs and participants can work together to determine which recording(s) would be most suitable or beneficial and that they listen to and engage in the exercises daily. (4) Physical domain recommendations will include $30 \mathrm{~min}$ of moderate intensity aerobic exercise (walking) and a program of six resistance training exercises to be performed 5 days per week. As part of the optional palette, participants with preoperative impaired pulmonary function will be recommended $15 \mathrm{~min}$ of daily inspiratory muscle training in the lead up to surgery.

\section{Safety and interactions review}

The safety and interactions review identified various scenarios in which certain NHPs are to be excluded from the intervention. All scenarios deemed absolute contraindications are given in Table 3 and include concurrent use of certain medications (e.g., green tea extract with nadolol), medical conditions (e.g., L-glutamine and seizure disorder), and allergies (e.g., fish oil and fish allergy). The safety and interactions review identified some areas of concern that will be highlighted in the study procedures document as situations where trial NDs should exercise clinical judgment regarding application of therapies (e.g., spacing whey protein away from medications including levodopa to avoid issues with absorption). Of note, individuals under 18 years of age, and pregnant and breastfeeding women will not be included in this study.

\section{Ethical and regulatory approval}

The pilot study that will evaluate the feasibility of the intervention has received ethical approval through the Research Ethics Boards at the Ottawa Health Science Network (20150449-01H) and the Canadian College of Naturopathic Medicine (CCNMREB019) and regulatory approval through Health Canada (HC6-24-c204361). The trial is registered through clinicaltrials.gov (NCT02845479).

\section{Discussion}

The pragmatic nature of the Thoracic POISE trial will allow us to assess integrative care in a manner that demonstrates model validity and reflects real-life routine practice conditions. ${ }^{25}$ Through a multistep, multidisciplinary process rooted in pragmatic, holistic, evidence driven, safe, feasible, and consensus-based principles, we have developed an integrative care intervention acceptable to all multidisciplinary stakeholders of the IDC, and within the scope of integrative oncology practices delivered by NDs. In Canada, the scope of naturopathic medicine is determined provincially in regulated provinces. The components of the integrative intervention palette are within the scope of NDs in all regulated Canadian provinces. In this article, transparent presentation of this process and its results are intended to support others seeking to develop and evaluate integrative care. Multiple aspects of this complex process merit further discussion.

The pragmatic nature of this trial presented a challenging aspect of the intervention selection process: the need for the intervention design to balance the requirements of replicable and standardized methodology to enhance the internal validity of the trial while still using a pragmatic approach reflecting the necessary and useful requirement of treating individual patient's symptoms. Our approach to achieve this balance was to design the intervention palette using a combination of standardized core components, supplemented with recommended optional components to give NDs the flexibility to choose therapies that best address an individual patient's needs (i.e., management of side effects during chemotherapy and radiotherapy).

The standardization of the intervention also allows the treatment to be implemented by other health care providers working in locations where an ND may not be available, allowing the results of the trial to be more broadly utilized should they be found to benefit patient outcomes. We also recognize that some integrative therapies in the physical, psychological, and nutritional domains are used in conventional care to some degree (e.g., exercise, ${ }^{26}$ diaphragmatic breathing, ${ }^{27}$ and nutrition counseling ${ }^{28}$ ). One distinction between integrative and conventional care is that conventional care providers are limited in the amount of time available to support patients in adopting these therapies, ${ }^{29}$ and interventions are often not studied in a holistic inclusive manner. The additional time spent with practitioners by the NDs in their care within this trial is indeed a component of the intervention and also reflective of its pragmatic nature.

The critical question to be addressed in the next stage of the Thoracic POISE trial is the feasibility of this integrative care intervention in a thoracic cancer population and the planned outcomes collection schedule. The planned singlearm Stage I feasibility study will allow us to refine the core 
and optional palettes and specific trial protocols before the RCT. This adaptive design is well suited for optimizing complex interventions, without compromising the internal validity of the planned randomized trial. The pilot stage will also help to minimize possible operational bias arising from modification of treatment for the final stage of the trial.

Valuable observations can be made from the results of the intervention development process. High degrees of variability were seen in both the scores assigned to components for evidence for effectiveness and in the level of concordance between scores for practical usage and evidence that further supports the need for rigorous research in this area. Some of the therapies with the highest level of practical usage (e.g., modified citrus pectin, arnica) have not been studied in thoracic cancer or other relevant surgical trials and we felt that their inclusion in this multiagent trial was thus not warranted. In contrast, some of the NHPs with the highest level of evidence for effectiveness (e.g., melatonin, L-arginine, Coriolus versicolor, astragalus, and probiotics) were selected by less than $55 \%$ of respondents in the ND practice survey. ${ }^{19}$ These findings may help to set priorities for research and knowledge translation efforts in the field of integrative oncology. Moreover, the multidisciplinary approach taken to define the integrative intervention palette provided a platform for communication and knowledge sharing between health care practitioners working in traditionally distinct settings.

Ultimately, the integrative care intervention palette we developed is but one iteration of a virtually endless number of permutations that could be used in this setting. This being the case, and without any pretense that this is the ideal intervention palette, the approach is nevertheless based on a pragmatic, rational, principle-driven process elucidated herein. Following results of the research, we will not know which of the components provide what outcome effect if indeed there are any. However, we are testing the ability of integrative medicine to make a difference in a variety of important health outcomes in a highly morbid condition and to the best of our ability make a meaningful difference for this population. The research will test in effect whether integrative medicine can make this difference and quantify this effect across various outcomes.

\section{Conclusion}

The Thoracic POISE trial will be the first pragmatic trial of its kind to explore the potential benefits of an integrative care intervention delivered by NDs for patients with resectable lung, gastric, and esophageal cancers. The process of defining the intervention palette in a systematic, transparent, and multidisciplinary manner and plan to assess the intervention rigorously will help to ultimately achieve the best possible design and implementation of the trial.

\section{Acknowledgments}

For their input as members of the intervention development committee, the authors thank: Lise Alschuler, Sylvain Boet, Chantal Bornais, Francesco Carli, Liane Feldman, Lorenzo Ferri, Christian Finley, Sylvain Gagne, Jeremy Grimshaw, Craig Herrington, Jelena Ivanovic, Scott Laurie, Robert MacRae, Penny Mariasine, Bill Nelems, Jason
Pantarotto, Susan Peter-Weeks, Lisa Rambout, Marisol Tiejeiro, Rebecca Van Schyndel. For funding of the Thoracic POISE project, the authors thank: The Lotte and John Hecht Memorial Foundation.

\section{Author Disclosure Statement}

No competing financial interests exist.

\section{Supplementary Material}

Supplementary Data

\section{References}

1. Jemal A, Siegel R, Ward E, et al. Thun MJ. Cancer Statistics, 2009. CA Cancer J Clin 2009;59:225-249.

2. Mitsudomi T, Suda K, Yatabe Y. Surgery for NSCLC in the era of personalized medicine. Nat Rev Clin Oncol 2013;10: $235-244$.

3. Lerut T, Moons J, Coosemans W, et al. Postoperative complications after transthoracic esophagectomy for cancer of the esophagus and gastroesophageal junction are correlated with early cancer recurrence. Ann Surg 2009;250: 798-807.

4. Fernandez FG, Kosinski AS, Furnary AP, et al. Differential effects of operative complications on survival after surgery for primary lung cancer. J Thorac Cardiovasc Surg 2017. DOI:10.1016/j.jtcvs.2017.09.149.

5. Kubota T, Hiki N, Sano T, et al. Prognostic significance of complications after curative surgery for gastric cancer. Ann Surg Oncol 2014;21:891-898.

6. Dziedzic DA, Rudzinski P, Langfort R, et al. risk factors for local and distant recurrence after surgical treatment in patients with non-small-cell lung cancer. Clin Lung Cancer 2016;17:e157-e167.

7. Spolverato G, Ejaz A, Kim Y, et al. Rates and patterns of recurrence after curative intent resection for gastric cancer: A United States Multi-Institutional Analysis. J Am Coll Surg 2014;219:664-675.

8. Lou F, Sima CS, Adusumilli PS, et al. Esophageal cancer recurrence patterns and implications for surveillance. J Thorac Oncol 2013;8:1558-1562.

9. Derogar M, Orsini N, Sadr-Azodi O, Lagergren P. Influence of major postoperative complications on health-related quality of life among long-term survivors of esophageal cancer surgery. J Clin Oncol 2012;30:1615-1619.

10. Khan NA, Quan H, Bugar JM, et al. Association of postoperative complications with hospital costs and length of stay in a tertiary care center. J Gen Intern Med 2006;21: 177-180.

11. Carey K, Stefos T, Shibei Zhao, et al. Excess costs attributable to postoperative complications. Med Care Res Rev 2011;68:490-503.

12. Vonlanthen R, Slankamenac K, Breitenstein S, et al. The impact of complications on costs of major surgical procedures. Ann Surg 2011;254:907-913.

13. Witt CM, Balneaves LG, Cardoso MJ, et al. A comprehensive definition for integrative oncology. JNCI Monogr 2017;2017. DOI:10.1093/jncimonographs/lgx012.

14. Horneber M, Bueschel G, Dennert G, et al. How many cancer patients use complementary and alternative medicine: A systematic review and meta-analysis. Integr Cancer Ther 2012;11:187-203.

15. Pratheeshkumar P, Sreekala C, Zhang Z, et al. Cancer prevention with promising natural products: Mechanisms of 
action and molecular targets. Anticancer Agents Med Chem 2012;12:1159-1184.

16. Seely D, Wu P, Fritz $\mathrm{H}$, et al. Melatonin as adjuvant cancer care with and without chemotherapy: A systematic review and meta-analysis of randomized trials. Integr Cancer Ther 2012;11:293-303.

17. Pitsouni E, Alexiou V, Saridakis V, et al. Does the use of probiotics/synbiotics prevent postoperative infections in patients undergoing abdominal surgery? A meta-analysis of randomized controlled trials. Eur J Clin Pharmacol 2009; 65:561-570.

18. Duncan M, Moschopoulou E, Herrington E, et al. Review of systematic reviews of non-pharmacological interventions to improve quality of life in cancer survivors. BMJ Open 2017;7:e015860.

19. Seely D, Ennis JK, McDonell E, Zhao L. Naturopathic oncology care for thoracic cancers: A practice survey. Integr Cancer Ther 2018;17:793-805.

20. U.S. National Library of Medicine. Search strategy used to create the complementary medicine subset on PubMed. Online document available at: https://www.nlm.nih.gov/bsd/ pubmed_subsets/comp_med_strategy.html, accessed January 28, 2019.

21. Willett W. Overview of nutritional epidemiology. In: Nutritional Epidemiology, 3rd ed. Oxford University Press, 2012. DOI:10.1093/acprof:oso/9780199754038.003.0001.

22. Seely D, Oneschuk D. Interactions of natural health products with biomedical cancer treatments. Curr Oncol 2008; 15 Suppl 2:s109.es81-s10.es6.
23. Health Canada. Listing of Monographs. Online document at: http://webprod.hc-sc.gc.ca/nhpid-bdipsn/monosReq.do\% 3Flang=eng accessed June 2, 2017.

24. Natural Medicines Comprehensive Database. About Natural Medicines Comprehensive Database. Online document at: http://naturaldatabase.therapeuticresearch.com, accessed June 2, 2017.

25. Patsopoulos NA. A pragmatic view on pragmatic trials. Dialogues Clin Neurosci 2011;13:217-224.

26. Pouwels S, Fiddelaers J, Teijink JAW, et al. Preoperative exercise therapy in lung surgery patients: A systematic review. Respir Med 2015;109:1495-1504.

27. Yates P, Schofield P, Zhao I, Currow D. Supportive and palliative care for lung cancer patients. J Thorac Dis 2013;5 Suppl 5:S623-S628.

28. Rock CL, Doyle C, Demark-Wahnefried W, et al. Nutrition and physical activity guidelines for cancer survivors. CA Cancer J Clin 2012;62:242-274.

29. Maizes V, Rakel D, Niemiec C. Integrative medicine and patient-centered care. Explor J Sci Heal 2009;5:277-289.

Address correspondence to: Dugald Seely, ND, MSc

Ottawa Integrative Cancer Centre 29 Bayswater Avenue Ottawa K1Y $42 E 5$ Canada

E-mail: dseely@oicc.ca 